\title{
Packet loss reduction function for multicast transmission on WLAN systems
}

\author{
Toshiyuki Ogawa ${ }^{1}$, Takefumi Hiraguri ${ }^{2 a)}$, \\ and Kenya Jin'no ${ }^{2}$ \\ ${ }^{1}$ Graduate School of Electrical Engineering, Nippon Institute of Technology \\ Gakuendai, Miyashiro-machi, Minamisaitama-gun, Saitama 345-85801, Japan \\ ${ }^{2}$ Department of Electrical and Electronics Engineering, Nippon Institute of \\ Technology
}

Gakuendai, Miyashiro-machi, Minamisaitama-gun, Saitama 345-85801, Japan

a) hira@nit.ac.jp

\begin{abstract}
This paper proposes an access control protocol method for multicast transmission to reduce packet loss caused by collisions and/or interferences in WLAN (wireless local area network) systems. Multicast transmission may achieve effective bandwidth use because packets are delivered to more than one mobile station simultaneously in single transmissions. However, because multicast transmission does not have a retransmission function, the communication quality deteriorates by packet collision and interference waves from other systems. The proposed method avoids both these issues. It is validated by a simulation showing multicast packet loss reduction of about $20 \%$ and a higher multicast throughput improvement compared to conventional methods, in an environment with traffic congestion and interference waves.
\end{abstract}

Keywords: WLAN system, multicast transmission, packets loss, packets collision, interference wave

Classification: Wireless Communication Technologies

\section{References}

[1] IEEE, "Part 11: Wireless LAN Medium Access Control (MAC) and Physical Layer (PHY) Specifications," IEEE Std 802.11 ${ }^{T M}-2007$, June 2007.

[2] A. Ganjam and H. Zhang, "Internet Multicast Video Delivery," Proc. IEEE, vol. 93, no. 1, Jan. 2005.

[3] M. T. Sun, L. Huang, A. Arora, and T. H. Lai, "Reliable MAC Layer Multicast in IEEE 802.11 Wireless Networks," Proc. IEEE ICPP, Aug. 2002.

[4] S. S. Byun and C. Yoo, "Fast Inter-Subnet Multicast Handoff Method for IEEE 802.11 WLANs," IEICE Trans. Commun., vol. E88-B, no. 8, Aug. 2005.

[5] OPNET [Online] http://www.opnet.com/ 


\section{Introduction}

IEEE802.11 WLAN (Wireless Local Area Network) systems [1] have been widely used in various applications and multimedia services. There is a video delivery method that uses multicast transmission for one of the multimedia services [2]. Because multicast transmission has the feature of group address delivery, a broadcasting service type that delivers the same contents to two or more wireless STAs (Stations) simultaneously can be achieved. Therefore, the multicast transmission may achieve an effective bandwidth use. However, there are some issues in video delivery using the broadcast type multicast in the WLAN. First, the transmission error due to interference waves from other systems is easily caused because the $2.4 \mathrm{GHz}$ bandwidth used by WLAN is an ISM (Industry Science Medical) band. Second, when the number of STAs increases and the bandwidth is saturated, packet collisions between the same systems are frequently generated if the same frequency is used. In particular, the collision immediately generates a packet loss in multicast transmission because this type of transmission does not have a retransmission function unlike the case of unicast transmission. Moreover, EDCA (Enhanced Distributed Channel Access) in IEEE802.11e [1] has a QoS (Quality of Service) function to support each application (VoIP and video, etc.); but, this is a function for unicast transmission and the QoS function suitable for multicast is not supported.

In this paper, we first describe the problems in conventional multicast transmission. Next, the proposed method to solve these problems is described. Finally, it is shown that the proposed method outperforms the conventional methods in terms of packet loss rate and throughput. The remainder of the paper is organized as follows. In section 2 , the conventional method and its problems are discussed. Section 3 describes the proposed method. Finally, section 4 shows via a computer simulation that the proposed method can reduce the packet loss rate and improve throughput performance compared with the conventional method.

\section{Conventional methods and their problems}

In the IEEE802.11 WLAN, a retransmission function is provided to minimize packet loss by retransmitting when the received STAs cannot receive the packet normally because of packet collisions and interference waves.

In unicast transmission, packet loss does not easily occur because the packets are restored by the retransmission function, as shown in Fig. 1 (a). However, multicast does not have the retransmission function. Therefore, the transmission error by packet collisions and/or interference wave immediately generates a packet loss, as shown in Fig. 1(b). EDCA performs the priority control by transmissions of different priorities that are appropriate for the application, such as video delivery, voice communication, etc. However, the priority control method suitable for multicast transmission has not been addressed adequately by the IEEE802.11e standard [3, 4]. The problem of multicast transmission will occur in environments with traffic congestion, when 
the priority control of EDCA is used experimentally, as shown in Fig. 1 (c). The consecutive transmission, called TXOP (Transmission Opportunity), is prepared as one of the access parameters to be given priority. Without interference wave, a collision is not caused during the TXOP period because TXOP reserves the bandwidth. However, the collision of an initial packet might be generated. With interference wave, packet loss will occur during the TXOP period because multicast packets are transmitted consecutively in each SIFS (Short Inter Frame Space). Therefore, SIFS does not perform the carrier sense.

To solve these problems, this paper proposes an access control method suitable for multicast transmission. The proposed method achieves high quality multicast transmission to avoid initial multicast packet collision and reduce the influence wave during the TXOP period.

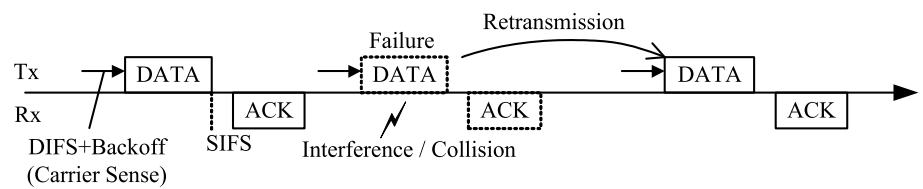

(a) Unicast transmission procedure

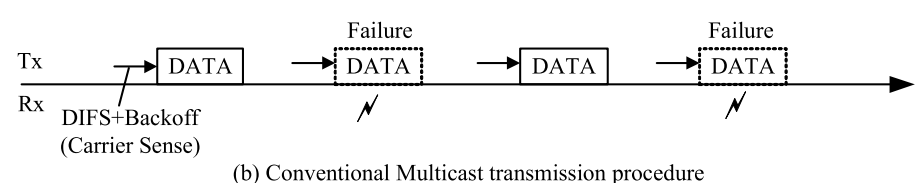

(b) Conventional Multicast transmission procedure

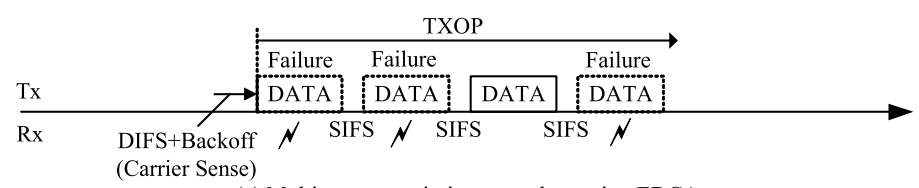

(c) Multicast transmission procedure using EDCA

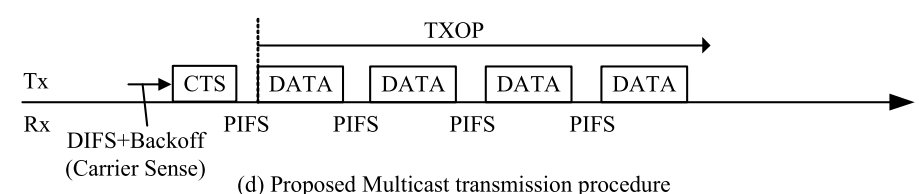

Fig. 1. Multicast transmission procedure with each method

\section{Proposed method}

The proposed method performs the carrier sense by DIFS (Distributed IFS) and backoff as well as a conventional method before the multicast transmission, as shown in Fig. 1(d). If the channel is idle, the frame that is called CTS (Clear to Send)-to-self is transmitted. The CTS-to-self frame is a control frame used in IEEE802.11g to exist together with IEEE802.11g and IEEE802.11b. The AP (access point)/STA that transmits CTS-to-self frame is allowed to occupy the bandwidth for the duration of information included in the CTS-to-self. In the proposed method, the CTS-to-self frame is used to avoid the collision of an initial multicast packet that the AP transmits. Moreover, the short carrier sense period of PIFS (Point IFS) is performed 
after the CTS-to-self frame is transmitted. Then, the multicast packet is transmitted if the channel is idle for PIFS. Afterwards, the multicast packet is transmitted continuously by the PIFS spacing until the TXOP period.

These functions are explained in Fig. 2. The example of Fig. 2 (a) is an operations sequence of the proposed method when AP acquires the transmission right at the same time as other STA. In this example, the CTS frame from AP causes the collision with the unicast packet from STA. After the collision, the unicast packet from STA is retransmitted according to a conventional control. On the other hand, AP detects the collision of the CTS-to-self if the channel is busy when the carrier sense of the PIFS period is performed. Then, AP retransmits the CTS-to-self frame again after DIFS and the backoff control. Avoiding the collision of the first multicast packet becomes possible by this control and the collision is with unicast data that is longer than the CTS-to-self frame; the loss of the following multicast packet can be prevented by the carrier sense at the PIFS period. Fig. 2 (b) is an operations sequence for the TXOP period when the interference wave arrives. To detect the interference wave, because the carrier sense of the PIFS period during the TXOP period continuous transmission is performed, multicast transmission will be stopped if the interference wave from other systems arrives while transmitting continuously. These functions will reduce multicast packets loss and the throughput will be improved.
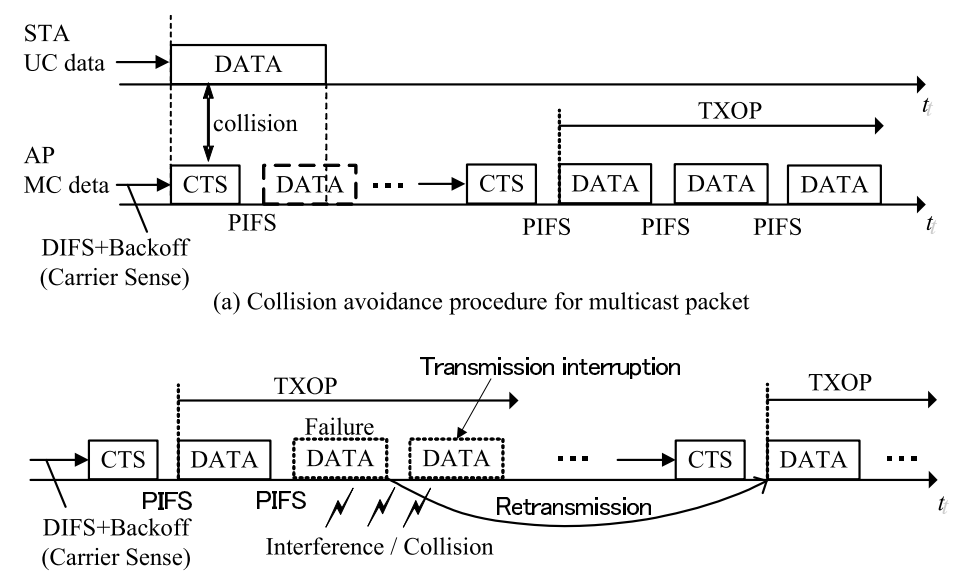

(b) Interference wave arrival detection and retransmission procedure for TXOP period

Fig. 2. Operations sequence of proposal method

\section{Simulation evaluation}

The packet loss rate and throughput characteristics of multicast were evaluated by computer simulation using OPNET Modeler v14.5 [5]. The network configuration in this simulation is described as follows. To evaluate the characteristics of the multicast, the delivery server of the multicast and the receiving terminal are prepared. The data of the delivery multicast is transmitted to the multicast receiving terminal through AP on the wireless network. To simulate the environment with frequent multicast packets collisions, ten STAs 
generating unicast traffic of the background load were connected to AP. The packet size of both multicast and unicast of the background load were set to 1500 bytes. The transmission link rate was set to $24 \mathrm{Mbit} / \mathrm{s}$ (mandatory maximum rate of IEEE802.11g standard). The packet loss and the throughput characteristics changed by the multicast traffic are shown in Fig. 3 (b) and (c), respectively. The horizontal axis shows the multicast offered traffic. The traffic amount of the background load STAs is assumed to be the same as in the multicast. For the conventional method, the packet loss outgrows it with an increase of traffic. The collision was frequently caused because of wireless bandwidth crowding. In this case, the packet loss increases similarly in the EDCA method. Moreover, the throughput of the conventional method has decreased greatly. The throughput is improved by the EDCA method. The throughput of the EDCA method is less than the offered traffic because of the influence of the packet loss. On the other hand, the proposed method reduced the packet loss tremendously, being almost absent. Moreover, the throughput of the proposed method was improved by about $10 \%$ compared with the EDCA method.

Next, to investigate the influence of a radio wave interference, the simu-

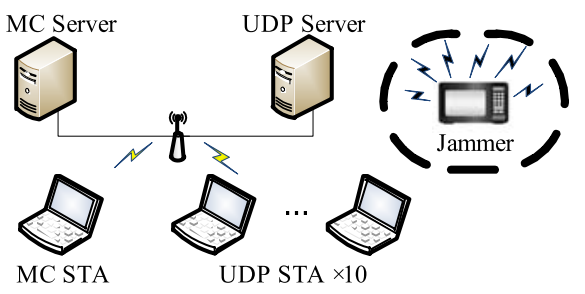

(a) Network configuration in simulation
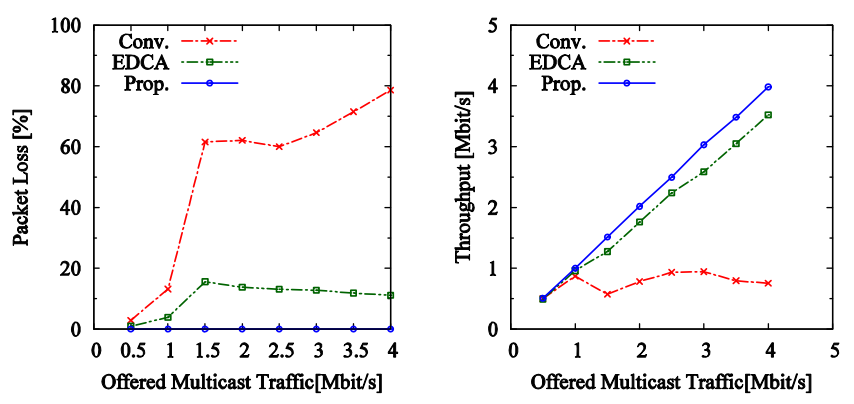

(b) Multicast traffic vs. packet loss

(c) Multicast traffic vs. throughput
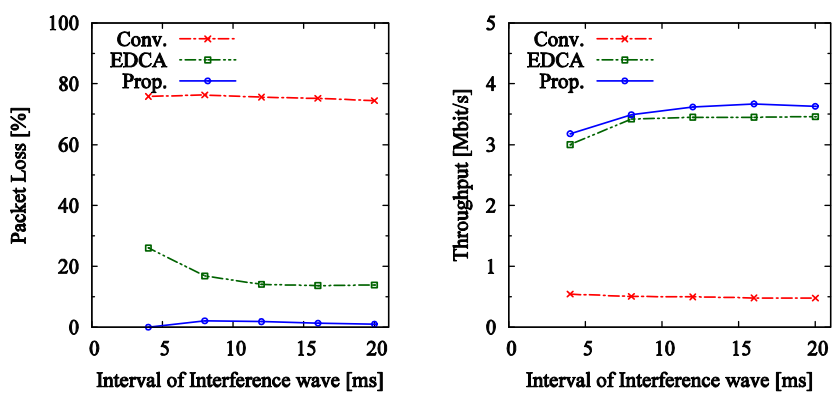

(d) Interval of interference wave vs. packet loss

(e) Interval of interference wave vs. throughput

Fig. 3. Packet loss and throughput by computer simulation. 
lation result when a jammer was added is shown in Fig. $3(\mathrm{~d})$ and (e). The jammer generates a rectangular waveform signal of the same frequency band. The signal generation interval of the jammer was changed from 4 to $20 \mathrm{~ms}$. The multicast and background load traffics from each STA generated 4 Mbps. The horizontal axis shows the interval of the jammer interference wave. In Fig. 3 (d), the packet loss rate of EDCA method increases by small intervals of the interference wave and packet loss is equal or greater than $20 \%$ when the interval of the interference wave is $4 \mathrm{~ms}$. Hence, the packet loss by the interference wave would be constantly caused while transmitting multicast. In the proposed method, the packet loss is substantially reduced even if the interference wave is generated. Moreover, the effect of throughput was higher than in EDCA, as shown in Fig. 3 (e).

\section{Conclusion}

This paper proposed a novel collision avoidance and retransmission functions for the interference wave in order to improve the quality of multicast. The key feature of the proposed method is its ability to avoid the collision of the multicast packet by transmitting the CTS-to-self frame first. The packet loss by the interference wave on continuous transmission during the TXOP period can be reduced by the carrier sense at the PIFS period. It was verified that the proposed method is able to considerably reduce packet loss by collision and interference waves, and improve the throughput more than traditional methods. 